\title{
Aggregation States and Proton Conductivity in Perfluorosulfonic Acid Thin Films under Humidity Condition
}

\author{
D. Kawaguchi ${ }^{1,2}$, S. Yonemori ${ }^{1}$, Y. Ogata ${ }^{1}$, N. Miyata ${ }^{3}$, T. Yoshida ${ }^{3}$, T. Miyazaki ${ }^{3}$, H. Aoki ${ }^{4,5}$, K. Tanaka $^{1,2,6}$ \\ ${ }^{1}$ Department of Applied Chemistry, ${ }^{2}$ Center for Polymer Interface and Molecular Adhesion Science, and ${ }^{6}$ International Institute for \\ Carbon-Neutral Energy Research (WPI-I2CNER), Kyushu University, 744 Motooka, Nishi-ku, Fukuoka, Japan, ${ }^{3}$ Neutron Science and \\ Technology Center, Comprehensive Research Organization for Science and Society, 162-1 Shirakata, Tokai, Ibaraki 319-1106, Japan, \\ ${ }^{4}$ Materials and Life Science Division, J-PARC Center, Japan Atomic Energy Agency, 2-4 Shirakata, Tokai, Ibaraki 319-1195, Japan \\ ,'Institute of Materials Structure Science, High Energy Accelerator Research Organization, 203-1 Shirakata, Tokai, Ibaraki 319-1106, \\ Japan. \\ d-kawaguchi@cstf.kyushu-u.ac.jp
}

In general, when a polymer film becomes thinner, the aggregation states and physical properties will deviate from those in the corresponding bulk due to surface and interfacial effects. This is also the case for perfluorosulfonic acid polyelectrolytes such as Nafion, Aquivion, etc. We previously reported that the anisotropic conductivity of protons was induced by thinning of a Nafion film in water.[1] This could be explained in terms of the peculiar aggregation states of Nafion close to the solid interface. In this study, the impact of the solid interface on the proton conductivity in Nafion thin films under a humidity condition was examined by alternating current (AC) impedance measurements in conjunction with neutron reflectivity (NR) measurements.

Nafion films were prepared by a spin-coating method from Nafion alcohol dispersions onto the substrates and dried under vacuum at $413 \mathrm{~K}$ for $3 \mathrm{~h}$. The films were kept under humidity condition for $5 \mathrm{~h}$ to reach an equilibrium swollen state. The AC impedance measurements were performed at room temperature by a two-point probe method with a Kelvin connection using an impedance analyzer combined with a micro-prober. The density profile of the Nafion film along the direction normal to the interface was examined by NR measurement under a $\mathrm{D}_{2} \mathrm{O}$ vapor condition (RH86\%) at $\mathrm{BL}-17$ in JPARC.

Figure 1 shows the thickness $\left(h_{\mathrm{w}}\right)$ dependence of in-plane proton conductivity $(\sigma)$ in Nafion thin films in water and under a humidity condition. While $\sigma$ increased with decreasing $h_{\mathrm{w}}$ in water, $\sigma$ decreased with decreasing $h_{\mathrm{w}}$ under the humidity condition. Since the interface-to-volume ratio increased with decreasing $h_{\mathrm{w}}$, it was evident that the thinning-induced $\sigma$ variation was due to an interfacial effect.

Panel (a) of Figure 2 represents an NR curve of the $57 \mathrm{~nm}$ thick Nafion thin film prepared on a quartz substrate under the $\mathrm{D}_{2} \mathrm{O}$ vapor condition. A solid line denotes the best-fit calculated reflectivity to the experimental one based on the model scattering length density $(b / V)$ profile shown in Figure 2(b). The model containing an interfacial segregation layer gave a better fitting for the experimental data. The interfacial layer having a lower $(b / V)$ value than the internal region may correspond to the initially adsorbed $\mathrm{H}_{2} \mathrm{O}$-contained one. This was in contrast to the interfacial structure of Nafion in $\mathrm{D}_{2} \mathrm{O}$, showing multi-layers with a total thickness of ca. $5 \mathrm{~nm}$ [1]. These results make it clear that the aggregation states of Nafion at a substrate interface were strongly affected by the wet environment. Thus, it can be concluded that the presence or absence of the interfacial multi-layers, or the two-dimensional proton-conductive pathway, enhanced and suppressed the in-plane proton conductivity.

[1] Ogata, Y. Abe, T., Yonemori, S., Yamada, N. L., Kawaguchi, D., Tanaka, K. (2018). Langmuir, 34, 15483-15489.

Keywords: Polyelectrolyte, Proton Conductivity, Neutron Reflectivity, Interface

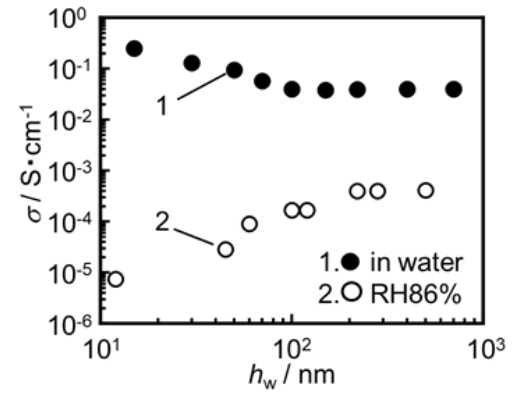

Fig. 1 Thickness $\left(h_{\mathrm{w}}\right)$ dependence of proton conductivity $(\sigma)$ in Nafion thin films on a quartz substrate along the in-plane direction in water and under humidity conditions at room temperature.
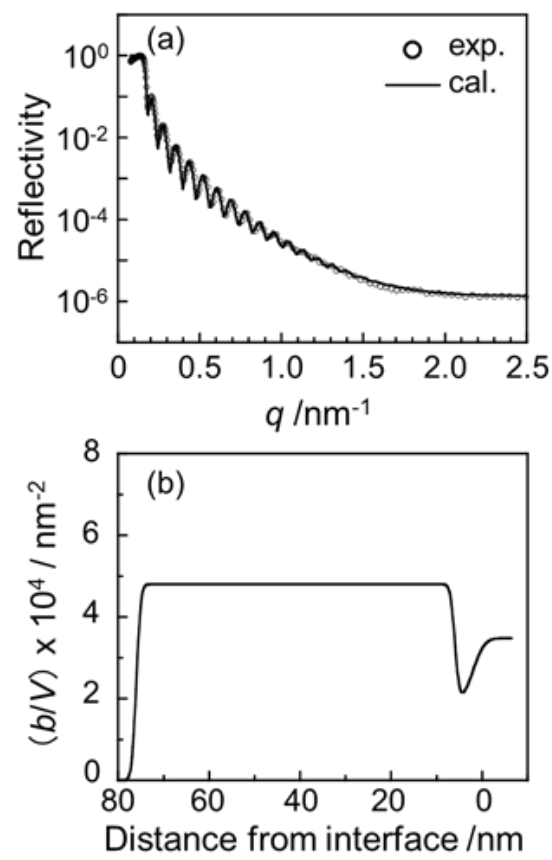

Fig. 2. (a) NR curve for a Nafion thin film supported on a quartz substrate under a $\mathrm{D}_{2} \mathrm{O}$ vapor condition at room temperature. Experimental data set is shown by open circles, and a best-fit curve calculated using the model $(b / V)$ profile shown in (b) is expressed by a solid line. 